\title{
UAV SYSTEMS FOR PHOTOGRAMMETRIC DATA ACQUISITION OF ARCHAEOLOGICAL SITES
}

\author{
M. Lo Brutto ${ }^{\text {a, } *}$, A. Borruso ${ }^{b}$, A. D’Argenio $^{\text {b }}$, \\ ${ }^{\text {a }}$ Dept. of Civil, Environmental, Aerospace and Materials Engineering, University of Palermo, Italy - mauro.lobrutto@unipa.it \\ ${ }^{\mathrm{b}}$ Consorzio Ticonzero, Palermo, Italy - (aborruso, adargenio)@ticonzero.net
}

KEY WORDS: UAVs, Photogrammetry, Archaeology, Computer Vision, DSM, Ortho image

\begin{abstract}
:
The use of UAV systems for surveying archaeological sites is becoming progressively more common due to the considerable potential in terms of rapidity of survey, costs and accuracy. The paper presents the first results of the photogrammetric survey of the archaeological site of Himera in Sicily (Italy) using by UAV systems. A complete documentation of the site through the production of a DSM and an ortho image were carried out. The research further evaluated two different image processing workflows: a typical photogrammetric approach and a computer vision approach. An ortho image of the archaeological site with a very high resolution was obtained.
\end{abstract}

\section{INTRODUCTION}

The documentation and preservation of archaeological sites often require the development of fast and easy techniques for 3D data acquisition, also in difficult conditions. Close range photogrammetry and terrestrial laser scanning are the most common techniques used. These techniques made it possible to obtain a high level of detail and accuracy and result to be very effective, especially for small or medium-extension archaeological sites (up to tens of hectares). However, for large archaeological sites close range photogrammetry and terrestrial laser scanner are not always the most suitable survey techniques; whereas, the information obtained from aerial or satellite images provide an overview of the study area, which is fundamental for the interpretation of archaeological structures. In fact, images obtained by metric aerial cameras (film and digital) or by high resolution satellite sensor have been used in archaeology for long (Cowley, 2011). It should be pointed out that such images have some limitations linked to the geometric resolution (typically of some decimetres and inadequate for detailed studies), to the periods of acquisition (which does not always correspond to a given particularly useful date for the purposes of the archaeological work), and ultimately to the cost. In the last years small UAVs (Unmanned Aerial Vehicles) have become standard platforms for large-scale aerial mapping of areas at limited extent. Many tests have been done to verify the photogrammetric aspects and their potential applications (Haala et al., 2011; Eisenbeiss \& Sauerbier, 2011). In particular, the performances of these photogrammetric systems are very high above all for aerial survey of archaeological sites (Eisenbeiss et al., 2005; Chiabrando et al., 2011; Hendrickx et al., 2011).

Beyond the acquisition phases, the image processing phase remains an unexplored topic as it should clearly defines the real capabilities of these systems for photogrammetric data collection and for archaeological survey.

In order to evaluate the UAVs workflow, some tests were performed for the study of the archaeological site of Himera (Sicily, southern Italy) using by close-range aerial photogrammetry techniques with micro UAVs. The main purpose was to obtain, besides the photographic documentation, a Digital Surface Model (DSM) and an ortho image of the site. The work was used to test different image processing workflows. The images were processed with both typical photogrammetric and computer vision approaches in order to identify the more efficient process. The two different approaches were compared in relation to the accuracy and automation of orientation and to the quality of photogrammetric data production.

The adopted pipeline was composed of different step like automatic flight planning, image orientation, image matching and DSM processing, ortho image generation.

The UAVs survey was allowed to acquire the data to extract a DSM and an ortho image of the site with a very high resolution and good accuracy.

\section{STUDY AREA}

The studied area corresponds to the higher part of the Himera archaeological site. The ancient city of Himera was situated on the coast of northern Sicily, about 30 kilometres far from Palermo. Himera, along with Selinunte, represents the western limit achieved by the Greek colonization. The first known settlement of Himera dates back to the VII Century BC; until its destruction (409 BC), the city extended to cover a surface of about 100 hectares.

The ancient urban area was built on a very complex landscape which included an area at the sea level (Lower Town) and a higher one (Upper Town); the Upper Town was located in a upland, named Himera Plane.

The work focused only on the Upper Town, where it is still possible to recognize two main groups of buildings: the "Northern Quarters" and the Sacred Area of the Athena Temenos. The "Northern Quarters" cover a surface of about four hectares and host the ruins of regularly shaped buildings; the Sacred Area of the Athena Temenos hosts four temples and is bounded by a perimeter wall.

\footnotetext{
* Corresponding author.
} 


\section{DATA ACQUISITION}

The aerial photogrammetric survey on the Himera site was realized using as carrier a microdrone md4-200 remotely piloted quadricopter. This carrier is equipped with four independent brushless electric motors and is able to fly up to 20 minutes at a maximum height of about $150 \mathrm{~m}$ (Table 1).

\begin{tabular}{|l|l|}
\hline Load capacity $(\mathrm{kg})$ & 0,2 \\
\hline Weight $(\mathrm{kg})$ & 0,9 \\
\hline Diameter $(\mathrm{cm})$ & 70 \\
\hline Operation range $(\mathrm{m})$ & 500 \\
\hline $\begin{array}{l}\text { Maximum relative } \\
\text { height }(\mathrm{m})\end{array}$ & 150 \\
\hline Flight range (min) & 20 \\
\hline Engines & $\begin{array}{l}4 \text { x 250W flatcore } \\
\text { brushless }\end{array}$ \\
\hline Camera & Pentax Optio A40 \\
\hline GPS & Yes \\
\hline Auto-pilot & Yes \\
\hline
\end{tabular}

Table 1: Technical specifications of md4-200

The quadricopter is radio controlled by a human operator, so the operation range is mostly limited by the reach of the radio control. Md4-200 electronic equipment includes an Inertial Measurement Unit (IMU), a GPS antenna and a communication system, which allows a ground based station to receive telemetry data from the carrier and video signals from the onboard sensors. Furthermore, an onboard Flight Controller device permits to autonomously execute automated flights. Automation includes route and all the issues related to image (or other kind of data) acquisition (such as: carrier attitude, camera orientation, zoom level). This feature is of particular interest in photogrammetric applications since it allows the design of flight plans with the desired forward overlap and side overlap. Thanks to the GPS antenna, flight planning can be designed on geospatial basis. This procedure can be executed in a software (mdCockpit) provided by the carrier manufacturer or, as in this case, in an external GIS environment. Owing to the low load capacity of the quadricopter, the system is equipped with a 12 Mpx compact consumer camera (Pentax Optio A40) slightly modified to be mounted on the bottom of the carrier.

The data acquisition workflow of the Himera site was composed by the following steps:

\footnotetext{
- $\quad$ Preliminary study of the area;

- $\quad$ Flight planning;

- Survey execution.
}

The "Preliminary study of the area" was subdivided in an identification of the site on cartography (mostly small scale maps and orthophotos), which was included in a GIS project, and an in situ inspection for the location of all possible problems for the flight (obstacles, physiography, electric cables, etc.).

For the "Flight planning" the archaeological site was divided in two parts: a larger area (about $135 \mathrm{~m} \times 285 \mathrm{~m}$ ), corresponding to the highest part of the ancient inhabited town (Upper Town) and a smaller one (about $35 \mathrm{~m} \times 60 \mathrm{~m}$ ), that includes the remains of the religious building of Athena Temenos. Owing to the limited autonomy of the drone, different photogrammetric flights were planned to cover the entire area. The Upper Town was covered by three flights at a relative height of $100 \mathrm{~m}$, while for the Athena Temenos was designed a single flight at $20 \mathrm{~m}$ from the ground surface (Figure 1). The "Flight planning" was performed in GIS environment and resulted in a code to be uploaded on the Flight Controller.

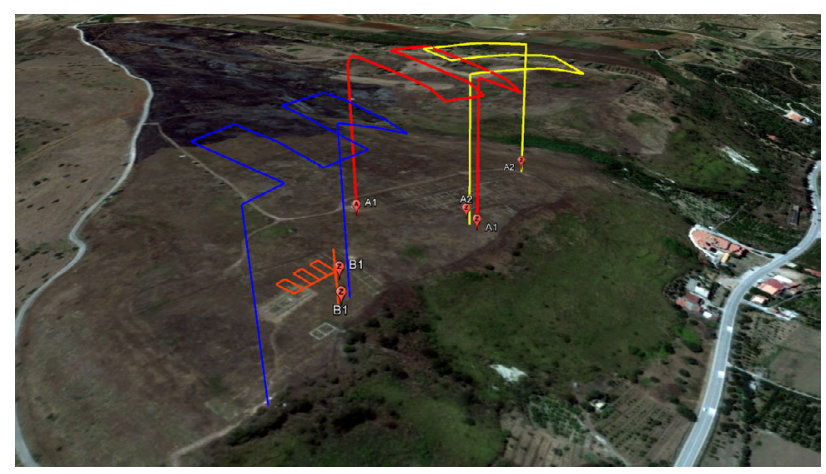

Figure 1: Flight planning

The images acquisition was executed setting the camera to its maximum resolution (12 Mpx) and using a focal length of 7.9 $\mathrm{mm}$ (Table 2).

\begin{tabular}{|c|c|}
\hline \multicolumn{2}{|c|}{ Pentax Optio A40 } \\
\hline Focal length & $7.9 \mathrm{~mm}$ \\
\hline CCD Width & $7.6 \mathrm{~mm}$ \\
\hline CCD Height & $5.7 \mathrm{~mm}$ \\
\hline Pixel size & $1.9 \mu \mathrm{m}$ \\
\hline Shutter time & $1 / 1000$ \\
\hline Aperture & 2.8 \\
\hline
\end{tabular}

Table 2: Camera parameters

With these parameters the Ground Sample Distance (GSD) was about $2.4 \mathrm{~cm}$, for the flights from $100 \mathrm{~m}$, and $0.4 \mathrm{~cm}$, for the flight from $20 \mathrm{~m}$. Image forward overlap and side overlap were set to $80 \%$ and $60 \%$, respectively (Table 3 ).

\begin{tabular}{|c|c|c|}
\hline \multirow{2}{*}{} & \multicolumn{2}{|c|}{ Flight height above ground } \\
\cline { 2 - 3 } & $100 \mathrm{~m}$ & $20 \mathrm{~m}$ \\
\hline Forward overlap & \multicolumn{2}{|c|}{$80 \%$} \\
\hline Side overlap & \multicolumn{2}{|c|}{$60 \%$} \\
\hline GSD & $2.4 \mathrm{~cm}$ & $0.4 \mathrm{~cm}$ \\
\hline $\begin{array}{c}\text { Ground coverage } \\
\text { per image }\end{array}$ & $96 \mathrm{~m} \mathrm{x} \mathrm{72} \mathrm{m}$ & $19 \mathrm{~m} \mathrm{x} \mathrm{14} \mathrm{m}$ \\
\hline
\end{tabular}

Table 3: Planned flight parameters

Regarding the "Survey execution", the flights were executed in autonomous mode, following the predefined flight path. This mode is the most useful for photogrammetric data acquisition. Images acquisition was performed in "stop mode"; the UAV system flies to a predefined way point and stops at its location to acquire the image (Eisenbeiss \& Sauerbier, 2011). For every way point three images were acquired to assure the adequate data redundancy. 
Before executing the flights, 30 targets, to be used as Ground Control Points (GCPs) during the image processing, were distributed along the area. A topographic survey was carried out to measure the coordinates of the targets using a Leica TCR 1105 total station. From a single point station, all the targets in a local reference system were measured. The accuracy of the target coordinates was estimated about $1 \div 2 \mathrm{~cm}$. Subsequently, two targets were measured additionally by a static GPS surveying with a Topcon Hyper Pro using as master station a permanent station of the University of Palermo GNSS Network. These two last points were used to convert the coordinates from the local reference system to global reference system WGS84ETRS2000 datum (UTM projection). The conversion was performed through a translation and a rotation of all the targets. No scale factor was applied during the coordinates computation to not introduce to the result all deformations of the cartographic projection. The cartographic height was obtained adding the difference between the height in the local reference system and the height in the WGS84-ETRS2000 datum calculated for one GPS point to all targets.

Flight execution lasted about two hours and produced 165 images at $100 \mathrm{~m}$ and 48 images at $20 \mathrm{~m}$.

\section{DATA PROCESSING}

The flights with micro UAVs are usually planned following the typical aerial flight where all images are acquired with a nadir view. This condition may not always be obtained with micro UAVs flight, because the short weight, the line and the attitude of flight can be modified by the wind.

The first phase of data processing was dedicated to the identification of the most suitable images for the work. In particular, it has been first verified the forward overlap and the side overlap. The figure 2 shows an example of a sequence of three images taken from the same waypoints; it can be noted that, even if the images were taken in a very short interval of time (few seconds), significant displacements of the camera may occur.

Besides, using consumer digital camera and in the absence of any device able to compensate for the movement of the sensor during the acquisition, the radiometric quality of the images can be degraded. This can affect both the automatic autocorrelation process and the manual photogrammetric measurement.

Therefore, the presence of images particularly blurred has been verified; the figure 3 shows a sequence of three images, taken by the same way point, where the image quality degrades considerably due to this effect.

The redundancy of images for each way point, however, has allowed the selection of a data set with the best characteristics as regards the block geometry and the radiometric quality. Overall 58 images were selected for flights from 100 meters and 17 images for the flight from 20 meters. Each flight from 100 meters was consisted of a photogrammetric block of three strips; two blocks had direction north-south, the third east-west. The flight from 20 meters had four strips and direction northsouth (Table 4).

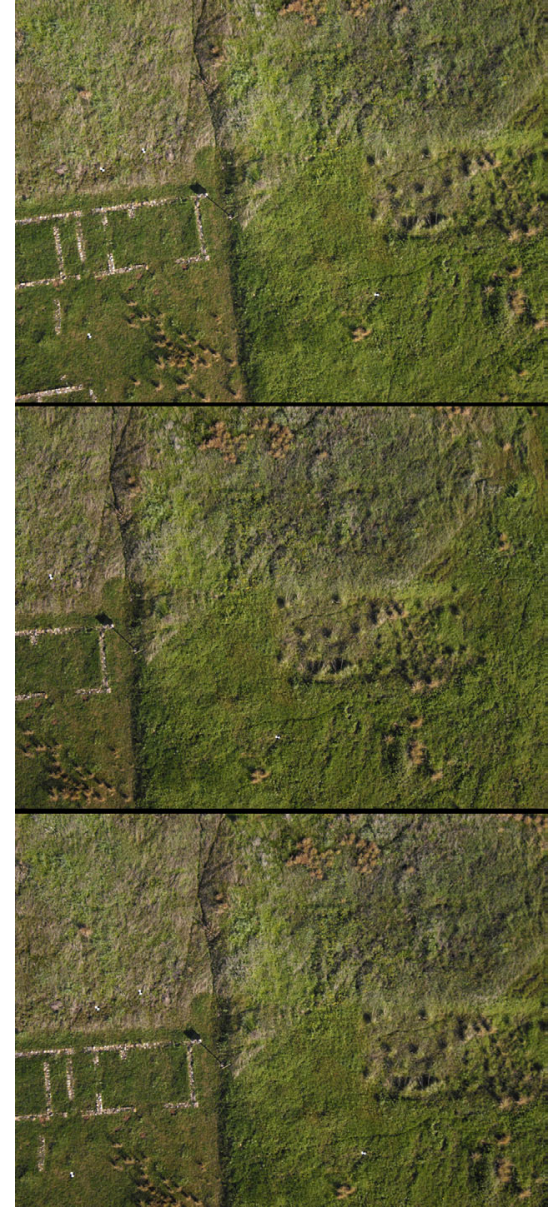

Figure 2: Sequence of three images taken from the same waypoints with significant displacements of the camera

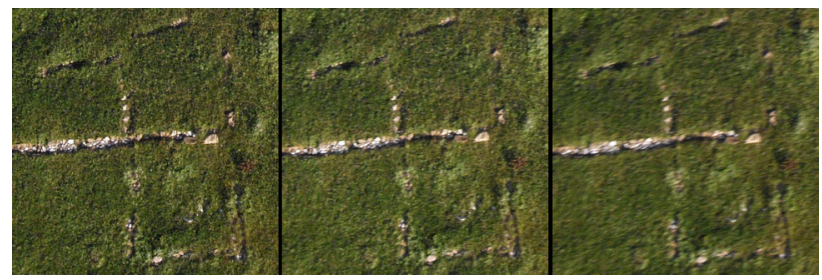

Figure 3: Sequence of three images taken by the same way point in which image quality degrades considerably.

\begin{tabular}{|c|c|c|c|c|}
\hline Flight & $\begin{array}{c}\text { Height } \\
\text { above } \\
\text { ground }\end{array}$ & Images & Strips & Direction \\
\hline F179 & $100 \mathrm{~m}$ & 23 & 3 & North-South \\
\hline F180 & $100 \mathrm{~m}$ & 20 & 3 & East-West \\
\hline F182 & $100 \mathrm{~m}$ & 15 & 3 & North-South \\
\hline F183 & $20 \mathrm{~m}$ & 17 & 4 & North-South \\
\hline
\end{tabular}

Table 4: Photogrammetric block configuration 


\subsection{Camera calibration}

A fully automatic self-calibration was carried out in laboratory using iWitness Pro software and 20 black \& white coded targets to determine the interior orientation parameters of the camera. The camera was set with the same parameters to be used during the flights: focal length fixed at minimum zoom (widest angle), focus fixed to infinite. The coded targets positioned to form a 3D calibration grid (Figure 4).

A network of 20 convergent images was taken from each side and from the diagonal of the grid. The network included also images with $\pm 90^{\circ}$ roll angles. Through the camera calibration the principal distance (c), the principal point position (xp, yp) and the radial distortion coefficients (K1, K2, K3) were calculated (Table 5).

For more accurate results, the camera calibration parameters should be obtained under conditions that are similar to the photogrammetric survey. This approach is not simple for UAV project because it requires a suitable test field and flight plan. Moreover, using consumer digital camera the overall accuracy obtained with lab calibration parameters and field calibration parameters are similar (Pérez et al. 2011).

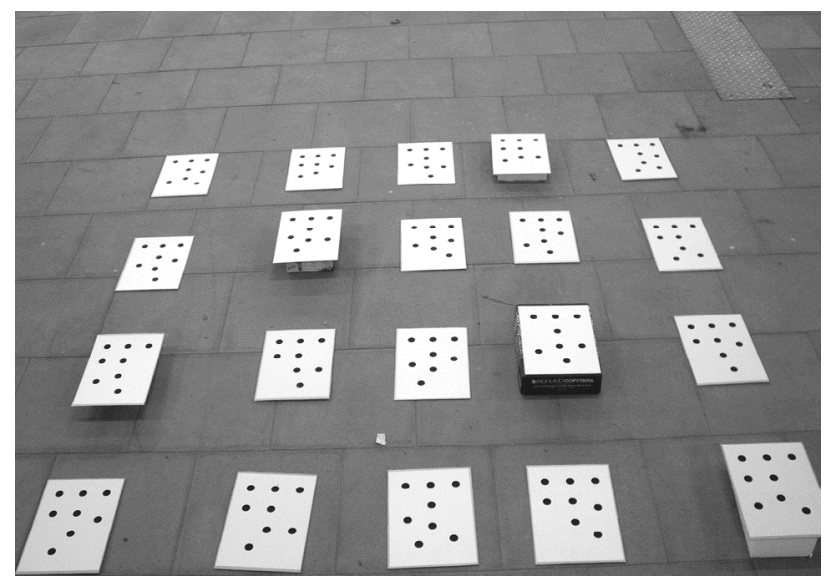

Figure 4: 3D calibration grid for camera calibration.

\begin{tabular}{|c|c|c|}
\hline Parameter & Value & Stand. dev. \\
\hline $\mathrm{c}$ & $8.765 \mathrm{~mm}$ & $0.001 \mathrm{~mm}$ \\
\hline $\mathrm{xp}$ & $-0.054 \mathrm{~mm}$ & $0.001 \mathrm{~mm}$ \\
\hline yp & $0.019 \mathrm{~mm}$ & $0.001 \mathrm{~mm}$ \\
\hline K1 & $2.07420 \mathrm{e}-003$ & $1.9049 \mathrm{e}-005$ \\
\hline K2 & $1.69550 \mathrm{e}-006$ & $2.4102 \mathrm{e}-007$ \\
\hline K3 & $-6.61121 \mathrm{e}-007$ & $9.1445 \mathrm{e}-008$ \\
\hline
\end{tabular}

Table 5: Camera calibration parameters.

\subsection{Images orientation}

In UAV projects, the images processing is still a topic of great interest as shown in several papers; not always the traditional photogrammetric systems are the most effective. Due to the various problems that may occur during the acquisition phase (irregular block geometry, poor radiometric quality) "not all software packages could be used in all possible applications of UAV photogrammetry; quite often, only the combination of several packages enabled us to completely process the data set" (Eisenbeiss, 2009). Some studies have been conducted to verify the possibility of using free and low cost software solutions (Neitzel et Klonowski, 2011), others to develop integrated procedures of computer vision and photogrammetry for fully automatic UAV image orientation (Barazzetti et al., 2010). In some applications, it was used an approach by the computer vision techniques to obtain orientation data and surface model to use as input for photogrammetric packages (Haala et al., 2011; Rosnell et al, 2011).

In this work the Flight F180 (Table 4) was used as dataset to test the different image orientation realized using two approaches: photogrammetric and computer vision techniques.

The photogrammetric orientation was carried out with the software Socet Set by BAE Systems; this package is one of the most popular software for the processing of aerial images.

For the photogrammetric image orientation the camera calibration parameters, previously calculated with iWitness Pro, were used. The approximate microdrone position and orientation data, provided by the GPS/IMU, were also used; in this way it was possible to reconstruct the photogrammetric block configuration.

An automatic aerial triangulation (AAT) was performed by the routine APM (Automatic Measurement Point) of the software Socet Set selecting a tie point pattern with 25 points per image distributed uniformly under a regular grid. Overall 776 image coordinates were measured obtaining automatically 82 tie points. The automated process required a phase of manual editing to correct some false matching. A quality check on some points showed that the poor stability of the microdrone produces images with very different perspectives of the same point (Figure 5).

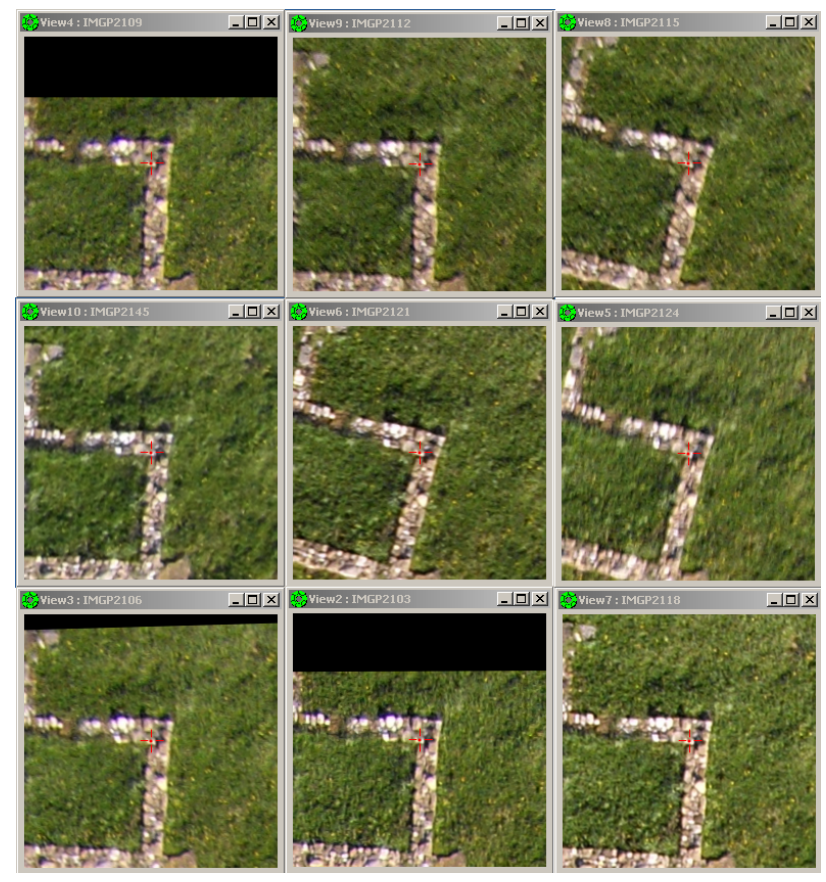

Figure 5: A tie point in several images

The exterior orientation parameters were computed using 8 GCPs; the control points were measured in semi-automated mode, identifying manually the target on an image and automatically by searching in all other through image matching. As shown in figure 6 the position of the control points was not suitable, however through a rigorous bundle block adjustment 
was possible to calculate the exterior orientation parameters obtaining a RMS for images residuals of \pm 1.4 pixel and for GCPs residuals of $\pm 4.7 \mathrm{~cm}, \pm 3.7 \mathrm{~cm}$ and $\pm 4.6 \mathrm{~cm}$ in the $X, Y$ and $\mathrm{Z}$ directions respectively.

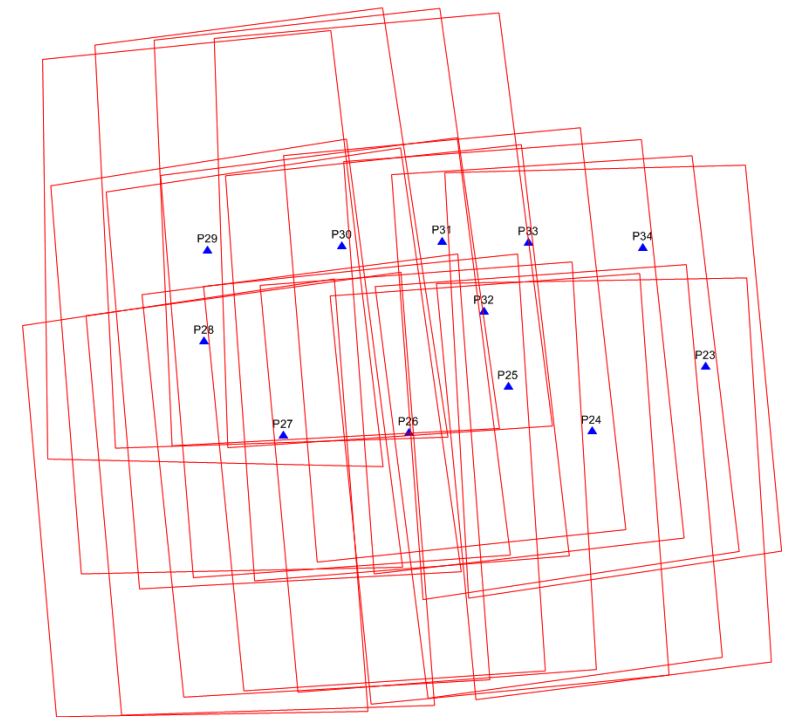

Figure 6: Block configuration and GCPs

Computer vision techniques was applied using the PhotoScan software (by Agisoft LLC) which is a low cost image-based package aimed to obtain high quality 3D model. The software is based on multi-view 3D reconstruction technology and can operate with calibrated and un-calibrated images in both controlled and uncontrolled conditions. The general workflow includes the fully automatic image orientation and 3D model reconstruction. All the processes can be performed with different levels of accuracy and many parameters can be set to improve the final result.

For lens calibration, PhotoScan software uses a pinhole camera model in the typical formulation of computer vision and, like all the computer vision applications, while carrying out photos alignment estimates both internal and external camera orientation parameters, including nonlinear radial distortions. For this reason, the camera calibration parameters previously calculated were not used.

With PhotoScan the automatic orientation was performed in various steps, with increasing accuracy. In a first step the images orientation was calculated with a low accuracy: the process oriented the 20 images of flight F180 in 4 minutes computing about 10000 tie points. Subsequently, the orientation was re-calculated with a medium accuracy obtaining about 54000 tie points in 7 minutes. Finally, the calculation was performed with the higher accuracy setting; a total of about 100000 tie points were determined in less than 10 minutes. The processing time, although with a much bigger number of points and with the higher accuracy setting, was much shorter than those obtained with the software Socet Set (about 15 minutes). It is important to note that the computation time depends on the PC characteristics, thus the previous information was reported to evidence the increase of computational load. In particular, for this work a PC with 32-bit Windows XP, a processor with 2.4 $\mathrm{GHz}$ and $4 \mathrm{~GB}$ of RAM was used. These characteristics, especially the 32-bit operation system, could affect the performance of the software.
The image orientation was obtained in a local reference system and in an arbitrary scale. The software do not provide any accuracy information about the image orientation. The camera parameters calculated during the self calibration were different in comparison with those obtained with lab calibration using iWitness Pro.

The photogrammetric targets were manually measured to transform the image orientation in the global reference system (WGS84-ETRS2000 datum - UTM projection). Assigning the cartographic coordinates to the targets, the photogrammetric block was referenced in the global reference system through a 3D transformation (3D translation, 3D rotation and a scale factor). Nine markers were used to compute the parameters of the $3 \mathrm{D}$ transformation obtaining a RMS of $\pm 2.5 \mathrm{~cm}, \pm 1.5 \mathrm{~cm}$ and $\pm 2.4 \mathrm{~cm}$ in the $\mathrm{X}, \mathrm{Y}$ and $\mathrm{Z}$ directions respectively.

\subsection{DSM and Ortho image generation}

A DSM and an ortho image were calculated with the two software after the images orientation.

With the software Socet Set the module NGATE, that performs image correlation and edge matching on each image pixel, was used. The DSM was calculated in a regular grid with a resolution of $50 \mathrm{~cm}$ by selecting the best images in relation to the radiometric characteristics and to their Base/Height ratio (Figure 7).

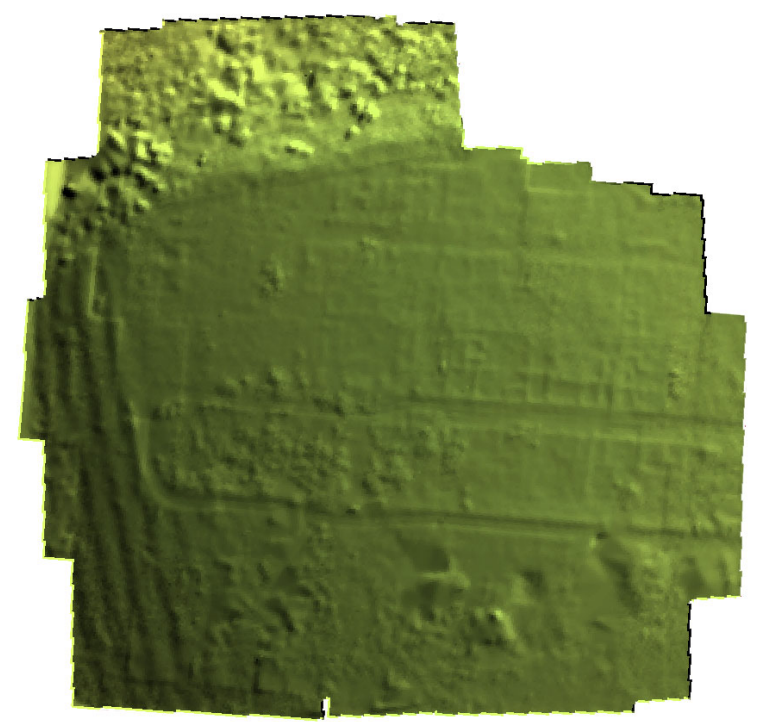

Figure 7: DSM obtained with Socet Set

Using the software PhotoScan it was not possible to define a geometric resolution of the DSM but the level of detail was chosen regarding the parameter "Target quality" (Ultra High, High, Medium, and Low, Lowest) and of the maximum number of faces in the final mesh. A "Target quality" medium and a maximum number of 200000 faces for the mesh were set for our work. The calculation of the DSM has allowed to obtain a surface with a point's resolution ranging from $20 \mathrm{~cm}$ to $60 \mathrm{~cm}$ (Figure 8).

With both software were made of the ortho images with a geometric resolution of $5 \mathrm{~cm}$. Only with Socet Set it was possible to select the best images for ortho image generation. A qualitative comparison between the two ortho images has showed no noticeable differences (Figure 9). 
For this reason, to obtain the data of the whole archaeological site, all the images were processed by computer vision approach with the software PhotoScan and the same workflow used for the flight F180. In this way processing time was significantly reduced. The figure 10 shows the ortho image obtained for the archaeological site of Himera by the flights from 100 meters.

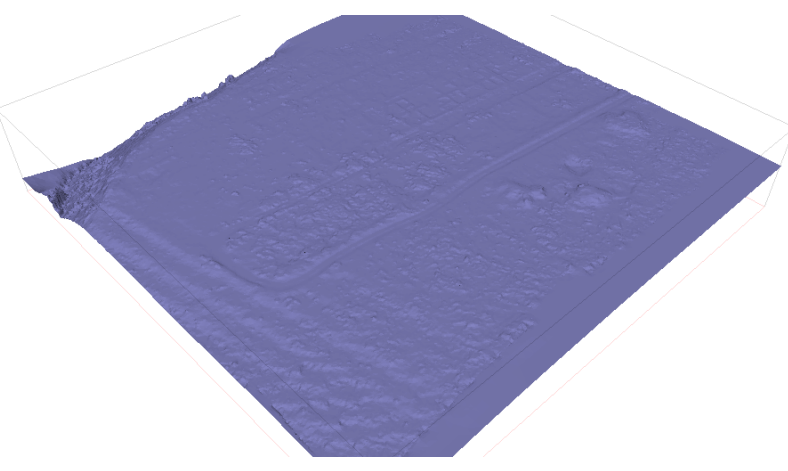

Figure 8: DSM obtained with PhotoScan

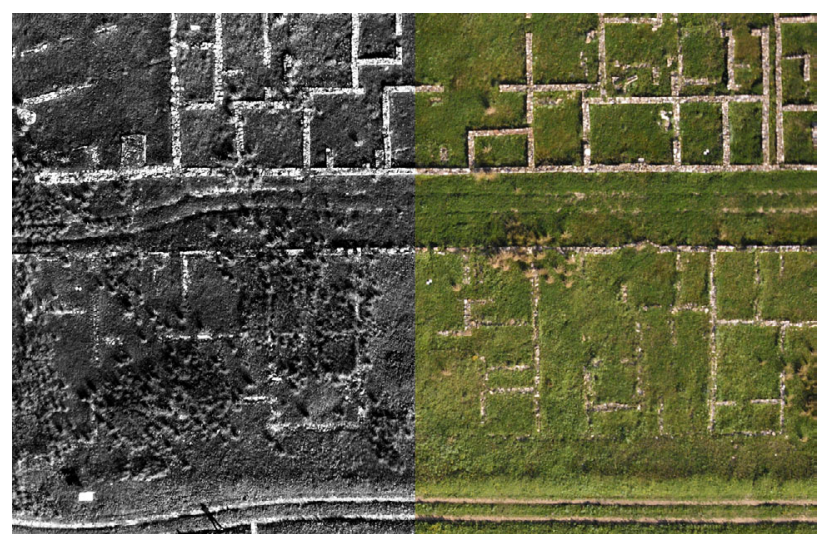

Figure 9: Visual check between the ortho images made with PhotoScan (in B\&W) and made with Socet Set (in RGB)

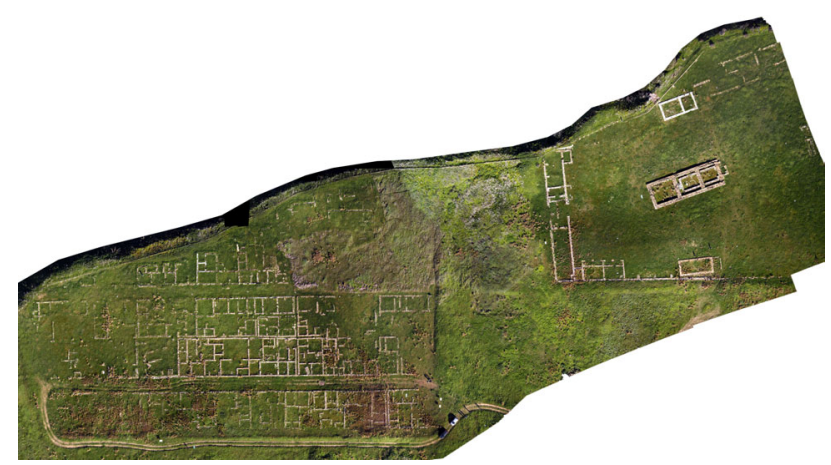

Figure 10: Ortho image of the archaeological site of Himera

\section{CONCLUSIONS}

The use of UAVs for the aerial survey of the archaeological site of Himera evidenced the high performance of these systems. Some problems could occur during image acquisition, however with a very high redundancy of images a DSM and an ortho image suitable for large scale mapping with very high resolution and accuracy can be obtained.
The computer vision technique has proven to be more simple and more fast than the photogrammetric one, though the last is a rigorous approach. For this reason the computer vision approach can be used on the survey of the archaeological sites; and further tests should to be performed in different locations with difficult morphological conditions to evaluate the real performance of UAV systems for rapid and accurate data acquisition.

\section{REFERENCES}

\section{References from Journals:}

Chiabrando, F., Nex, F., Piatti, D., Rinaudo, F., 2011. UAV and RPV systems for photogrammetric surveys in archaeological areas: two tests in the Piedmont region (Italy). Journal of Archaeological Science, 38(3), pp. 697-710.

Eisenbeiss, H., Sauerbier, M., 2011. Investigation of UAV systems and flight modes for photogrammetric applications. The Photogrammetric Record, 26(136), pp. 400-421.

Hendrickx, M., Gheyle, W., Bonne, J., Bourgeois, J., De Wulf, A., Goossens, R., 2011. The use of stereoscopic images taken from a microdrone for the documentation of heritage - An example from the Tuekta burial mounds in the Russian Altay. Journal of Archaeological Science, 38(11), pp. 2968-2978.

\section{References from Other Literature:}

Barazzetti, L., Remondino, F., Scaioni, M., 2010. Fully automated UAV image-based sensor orientation. International Archives of the Photogrammetry, Remote Sensing and Spatial Information Sciences, Vol. XXXVIII-1, p. 6.

Cowley, D.C., 2011. Remote Sensing for Archaeological Heritage Management. EAC Occasional Paper No. 5; Occasional Publication of the Aerial Archaeology Research Group No. 3; Europae Archaeologiae Consilium: Budapest, Hungary, p. 307.

Eisenbeiss, H., 2009. UAV photogrammetry. Diss. ETH No. 18515, Institute of Geodesy and Photogrammetry, ETH Zurich, Switzerland, Mitteilungen Nr.105, p. 235.

Eisenbeiss, H., Lambers, K., Sauerbier, M., Zhang, L., 2005. Photogrammetric documentation of an archaeological site (Palpa, Peru) using an autonomous model helicopter. International Archives of the Photogrammetry, Remote Sensing and Spatial Information Sciences, Vol. XXXIV-5/C34, pp. 238243.

Haala, N., Cramer, M., Weimer, F., Trittler, M., 2011. Performance test on UAV-based photogrammetric data collection. International Archives of the Photogrammetry, Remote Sensing and Spatial Information Sciences, Vol. XXXVIII-1/C22, p. 6.

Neitzel, F., Klonowski, J., 2011. Mobile 3D mapping with a low cost UAV system. International Archives of the Photogrammetry, Remote Sensing and Spatial Information Sciences, Vol. XXXVIII-1/C22, p. 6.

Pérez, M., Aguera, F., Carvajal, F., 2011. Digital camera calibration using images taken from an unmanned aerial vehicle. International Archives of the Photogrammetry, Remote Sensing and Spatial Information Sciences, Vol. XXXVIII1/C22, p. 6. 
Rosnell, T., Honkavaara, E., Nurminen, K., 2011. On geometric processing of multi-temporal image data collected by light UAV systems. International Archives of the Photogrammetry, Remote Sensing and Spatial Information Sciences, Vol. XXXVIII-1/C22, p. 6 
